\title{
MRI and CT of the Female Pelvis
}

\author{
B. Hamm and R. Forstner, eds.
}

New York, NY: Springer, 2007, 388 pages, $\$ 219$

One of the most challenging problems in clinical practice is discerning the cause of pelvic pain. Cross-sectional imaging contributes important information for proper diagnosis of gynecologic diseases and for therapeutic decisions regarding them and thus plays an ever-increasing role in diagnostic and interventional studies of the female pelvis. Cross-sectional imaging has been used to determine the stage of pelvic tumors and the extent of pelvic diseases. MRI has been the imaging modality of first choice for dedicated examinations of the female pelvis since early in 1990, and modern techniques are used for the diagnosis of pelvic floor or sphincter disorders. Continuous technical progress in MRI and CT required the need for a volume dedicated to these modalities in the book series Medical Radiology: Diagnostic Imaging, edited by A.L. Baert, M. Knauth, and K. Sartor. The 17 European and 1 American contributors are acknowledged experts in diagnostic imaging of the female pelvis. The editors selected the authors of individual chapters on the basis of their broad experience in and personal knowledge of specific pathologic disorders, and the authors have written their contributions according to their own approaches, with current and updated information.

This book is organized into 17 chapters dealing with anatomy; MRI and CT techniques; normal findings for the uterus; uterine malformations; benign uterine lesions; endometrial cancer; cervical cancer; normal findings, anomalies, and benign and malignant lesions of the ovary; endometriosis; the vagina; pelvic floor MRI; MRI pelvimetry; imaging of lymph nodes; infertility; and pelvic pain disorders. The main emphasis is placed on the most recent diagnostic and technical advances, and the book is complemented by many detailed and informative illustrations, which are excellent. The chapters on the various disorders are preceded by information on pelvic anatomy and state-of-the-art MRI and CT techniques that characterize developmental anomalies and acquired diseases. Benign and malignant diseases are considered in depth and with detailed attention. Chapters on pelvic pain and MRI pelvimetry are helpful in the evaluation of pregnancy and fertility. Besides presenting a kind of cookbook with tips on practical imaging strategies, this book is easy to read and provides clear guidelines on indications, differential diagnoses, and the pitfalls of MRI and $\mathrm{CT}$.

This book is a comprehensive, detailed, up-to-date review of current knowledge on using MRI and CT for the fascinating diagnosis of female pelvic disorders. The book will be of great value to radiologists, nuclear physicians, obstetricians, gynecologists, radiation oncologists, urologists, general surgeons, and family physicians in training and practice. It will also be useful to all interested in MRI and CT of the female pelvis, and our patients will benefit from optimal use of these diagnostic tools.
E. Edmund Kim M.D. Anderson Cancer Center Houston, Texas

COPYRIGHT (C) 2008 by the Society of Nuclear Medicine, Inc.

DOI: 10.2967/jnumed.107.050229 